\title{
Leakage Detection in Pipeline using Wavelet Transform Method
}

\author{
M.F Ghazali ${ }^{1}$, A. K Samta ${ }^{1}$ \\ ${ }^{1}$ Faculty of Mechanical Engineering, Universiti Malaysia Pahang, Malaysia \\ Email:fairusham@ump.edu.my
}

\begin{abstract}
This research project is focusing on the leakage detection in the pipelines system using wavelet transform method. The signal contains information to whether a leak exists and where it is located. Pressure transient happened when there is a sudden change in flow by closing or opening the valve in the pipelines. Such that wave propagation created along the pipelines, and this propagation will be used as a medium to detect the leaks. The materials used for the experimental analysis are high-density polyethylene (MDPE) and Galvanized iron (GI) pipes. In order to filter noise from the signals, the discrete wavelet transform method is used in this research. In order to collect the data, three type experiments have been performed by using two different pipe material. They are open one leak, two leak, and both leaks. The experimental results show the ability of the method to identify features in the signal.
\end{abstract}

Keywords leakage; pipelines; pressure wave; wavelet transform

Paper type Research paper

\section{INTRODUCTION}

Water loss is a common problem that occurs in all water supply system. Most urban areas throughout the world are losing 30 to $50 \%$ of their water supply to leakage. An investment in repairing leaks, purchasing new pipes and maintaining existing and new pipe structures would pay for itself in conserved water in a few years. For example, Mexico City's water system loses 1.9 billion cubic meters of water every year due to leakage [1].

A significant part of the recent literature concerns the application of hydraulic transients in leak detection. For example, some previous studies deal specifically with the use of transient analysis. For example, Brunone [2] and Brunone and Ferrante [3], utilize the approach based on the detection and location of leak reflected pressure wave in the transient signal. While simple to apply, background noise can complicate the leak reflection signals. As with Beck et al. [4] exploited the disturbance in the pressure signal caused by the leak and used an extension of cross-correlation analysis techniques to locate the position of a leak.

Ferrante et al. [3] use wavelet analysis to detect local singularities due to the presence of a leak in the measured transient signal. Al-Shidhani et al. [5] also performed wavelet transforms on experimental and simulated transients in pipe systems. The technique identified singularities in the simulated data; however, when the same analysis was applied to the experimental data, the expected results did not agree with the lengths found in the network. More recently, many engineers and researchers interested in using water hammer analysis for leak detection techniques [6]. This method used pressure waves which are called pressure transient causes by quickly opening and closing a valve and known as water hammer that a pressure fluctuation in the pipeline due to a change in the system. When pressure transient create the wave encounters features of test rig such as a leak or connection, the additional points can create and measured by using a single pressure transducer. The signal analysis work reported the method of analysis transient signal by using a wavelet transform method so that it provides more useful information about the instantaneous frequency (IF) [7]. The main objective of using the wavelet analysis is to filter and remove noise from the data to detect leakage in the pipeline system.

\section{WAVELET}

Leakage in the pipelines is becoming a huge issue in the world. Quick and smart action should be taken in order to minimize the loss and effect of the leakage. A traditional method such as visual inspection can be operated but that method required a long time. It is also a lack of accuracy and may lead to false leak detection. Through signal analysis, the leak can be detected, and the location of the leaks can be located. So, without the need for another method, the leaks can be repaired in no time. Signal analysis is a method of analyzing the data from the piping system that can be obtained through a sensor installed at the pipe.

The history of signal analysis is starting by using Fourier transform techniques. However, this method has its limitations. It can only detect the leaks but cannot identify the location and the time when the leaks occurred [6], [7]. So in 
order to obtain an accurate and complete data of the leak, many techniques of signal analysis have been developed such as wavelet, cepstrum, empirical mode decomposition (EMD) and many more.

The wavelet transform is the most recent or newest solution to overcome the shortcomings of the Fourier transform. This method uses a fully scalable modulated window to solves the signal-cutting problem. The window is shifted along the signal, and for every position, the spectrum is calculated. Then this process is repeated many times with a slightly shorter or longer window for every new cycle. In the case of wavelets, usually do not speak about time-frequency representations but about time-scale representations, the scale being in a way the opposite of frequency, because the term frequency is reserved for the Fourier transform [8]. Wavelet can be categorized into two types which are continuous wavelet transform and discrete wavelet transform

The continuous wavelet transform is defined as the sum over all time of the signal multiplied by a scaled, shifted version of the wavelets function where both the time and frequency windows can be changed. The idea of this method is generally the same as the Short Time Fourier Transform (STFT), but the only difference is that the width of the windows is continuously changed throughout the transform. Theoretically, it can be calculated through the equation below:

$$
Q(a, b)=\frac{1}{\sqrt{\alpha}} \int_{-\infty}^{\infty} y(t) \varphi^{*}\left(\frac{t-b}{a}\right) d t
$$

Where the index $Q(a, b)=$ wavelet coefficients, $a$ and $b$ are the scale (dilation) and translation(position) parameters, $y(t)=$ vibration response signal, $\varphi^{*}$ is the complex conjugate of the basis function. Disadvantages of continuous wavelet transform are this method consumes longer time and more resources to operate.

\section{EXPERIMENTAL DESIGN}

The transient method will be used in this study. This method is one of the commonly used techniques in the leakage detection method [10]. It is the idea of using transient pressure flow. Pressure transient happened when there is a sudden change in flow by closing or opening the valve in the pipelines. Such that wave propagation created along the pipelines, and this propagation will be used as a medium to detect the leaks. The materials used for the experimental analysis are high-density polyethylene (MDPE) and Galvanized iron (GI) pipes. The GI pipe is also a popular method of plumbing water supply in the home, but it gradually fails due to corrosion and rust. It is no longer commonly used and has been replaced with HDPE pipe. Also, this type of pipe is hard to cut and join and not easily fabricated on site by the homeowner. However, this type of pipes is cheap, light in weight and easy to handle transport and easy to join [11].

\section{Preparing THe TeSt Rig}

The experimental tests were conducted using a pipeline network as shown in the schematic diagram below. The pipes used are HDPE and GI pipe with diameter 1 inch and half an inch. The inlet of the pipeline is connected to the water tank to supply constant water pressure in the system. The effect of leaks into the system, pinhole that controlled by valve has been installed at various location along with the pipeline network. A solenoid valve is used to maintain pressure in the test rig system. The signal is captured using the pressure transducer located near the solenoid valve. The pressure transducer is connected to the data acquisition system. Each run lasted for 1 minute and 10 seconds. In order to filter noise from the signals, the discrete wavelet transform method is used in this research. Figure 1 shows the design of the test rig pipeline network.

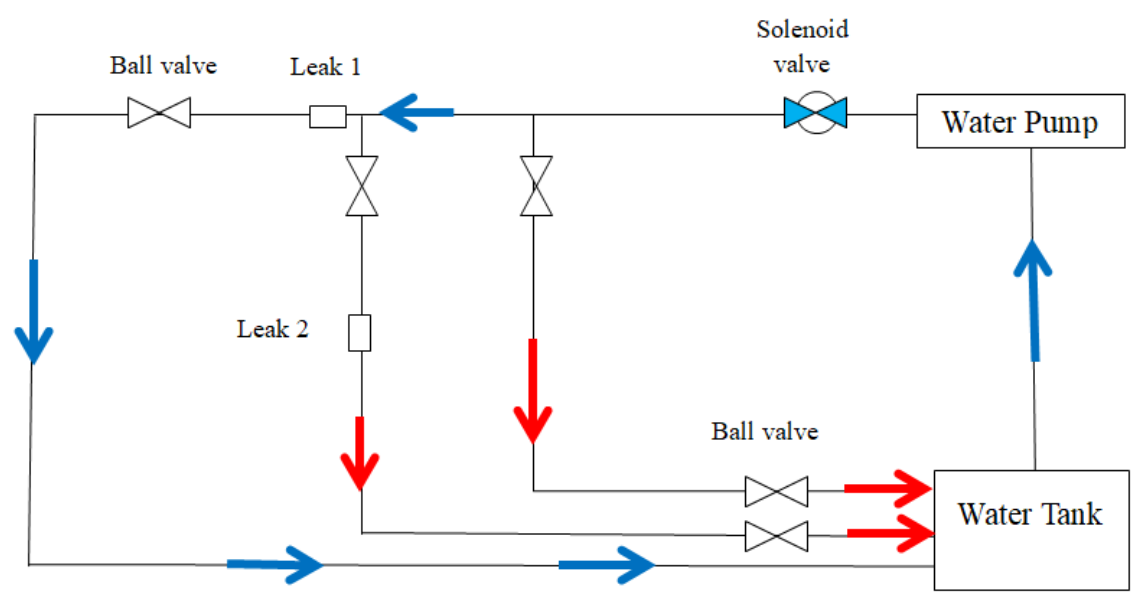

Fig. 1. The test rig pipeline network. 


\section{RESULT AND DISCUSSION}

Data is collected by using two sensors which are strain gauge pressure transducers and Piezotronics pressure sensor. Two different material used in the test rig is galvanized iron (GI) and Medium Density Polyethylene (MDPE) pipes. For each material, two trial of data is collected and for every different trial position of features is included such as opening both leaks, opening the only leak one, open only leak two and no leak open. The samples size and sampling rate used for the experiment are 6400 for GI pipe and 1000 for MDPE pipe. When raw data is collected, the wavelet transforms analysis will first take place. The data will be a load on the wavelet toolbox of one dimensional in the MATLAB. Figure 2 shows the sampled data. The type of wavelet uses to decompose the signal is $\mathrm{db}-3$ with five levels, because of its compatibility to the raw signal which looks like the noise mishmash. The main objective of using the wavelet analysis is to filter and remove noise from the data. Only when the noise from the data which is not needed is removed, such that the signal of the features can be seen clearly.

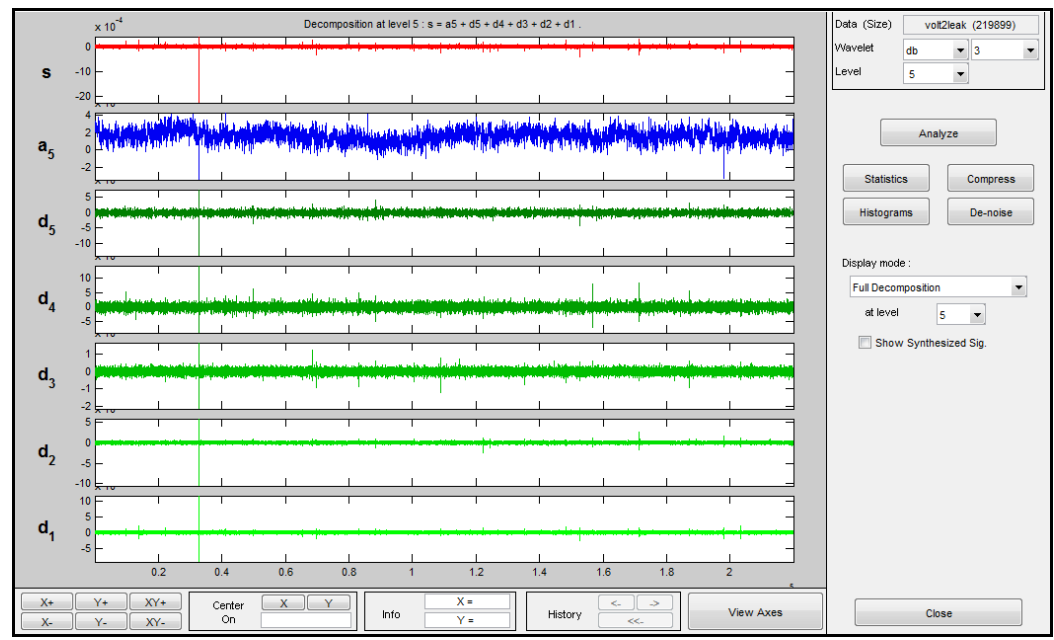

Fig. 2. Sampled data of wavelet filter and decompose

The process of removing the noise is called the de-noising process. It is performed by selecting the scaled white noise which exists in the data due to pump noise, surrounding noise and many other disturbances. Figure 3 shows the selection of scaled white noise for a de-noising process, where Figures 3-6 shows the original signal, de-noised signal, and comparison between original and de-noised signal. Based on the figures, it can be seen that there is a difference from before and after de-noised. The signal that is needed is seen on the graph after being de-noised. However, the process of filtering and de-noising depends on the amount of noise in the raw data.

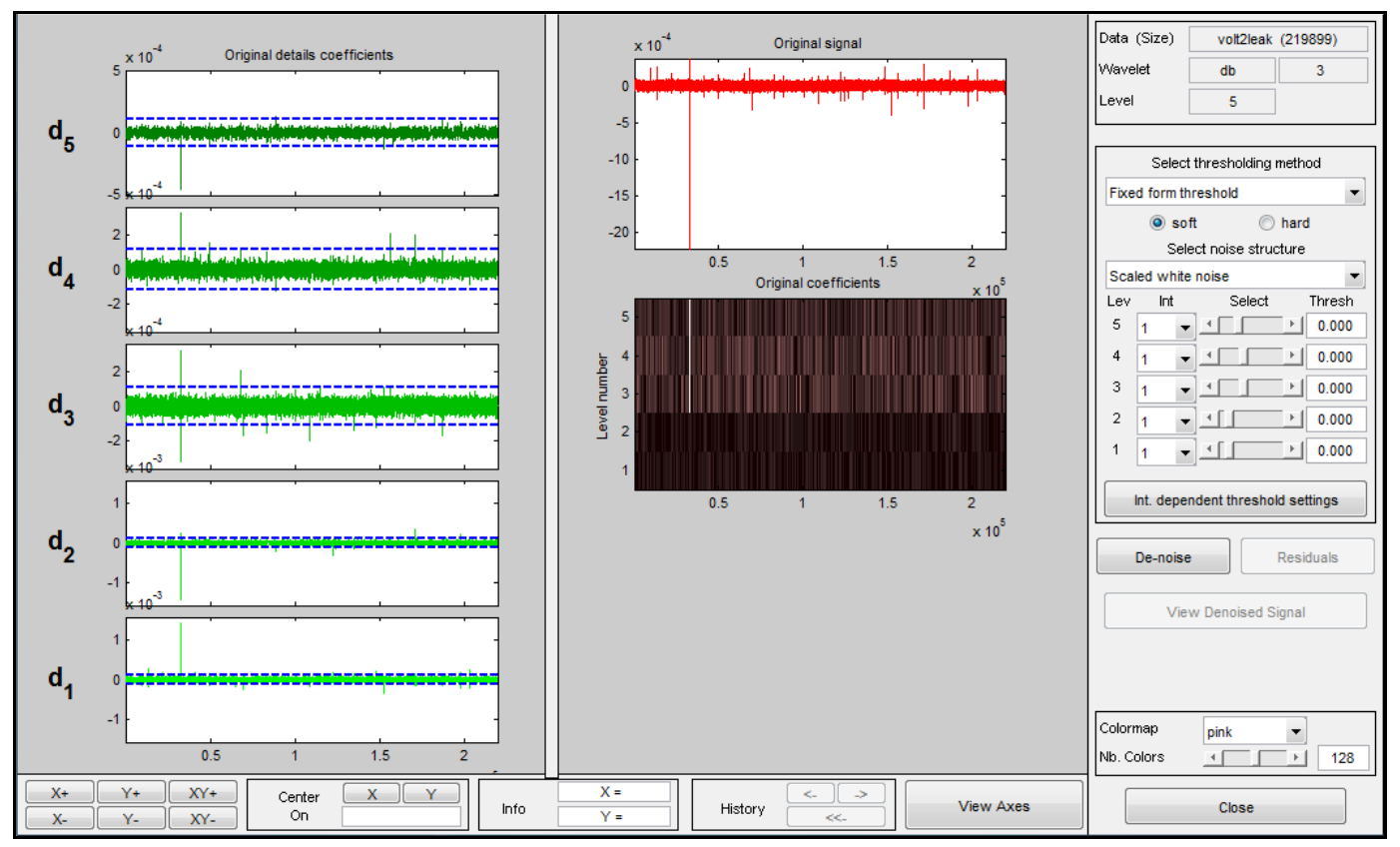

Fig. 3. Sampled data for de-noising 


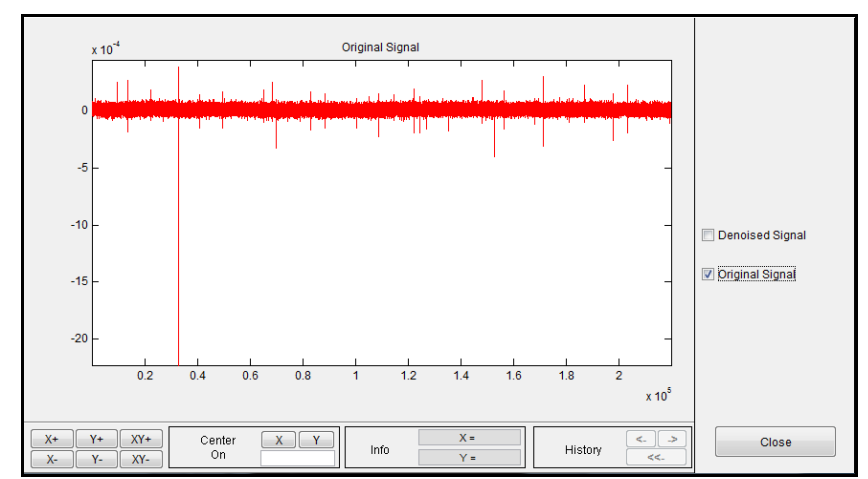

Fig. 4. Original signal

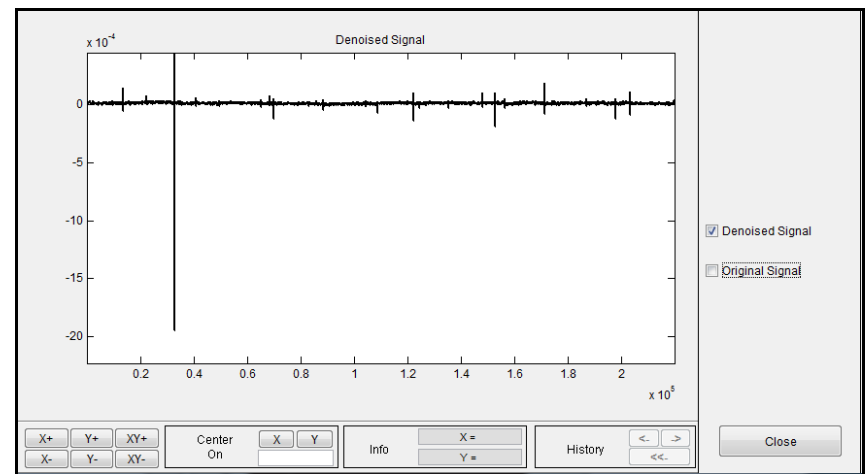

Fig. 5. De-noised signal

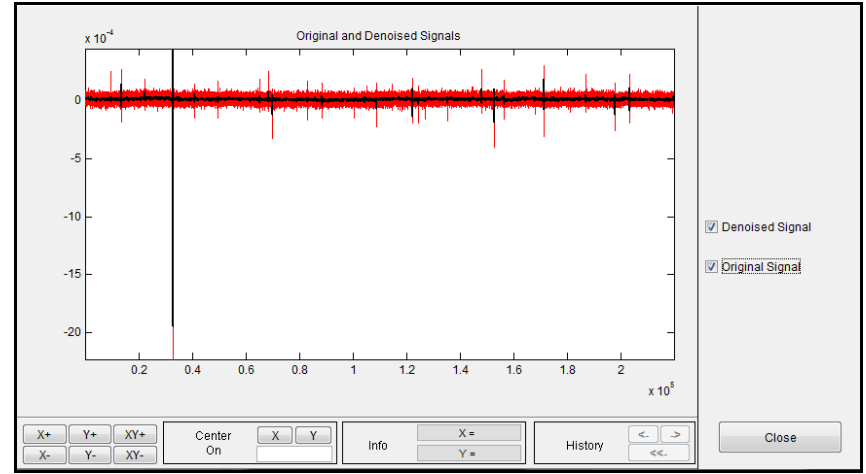

Fig. 6. Comparison between the original and the de-noised signal

The first material used for the experiment is the galvanized iron pipe. In order to collect the data, two trial experiments have been performed by using this material, and for every trial, three different conditions of features are tested. Theoretically, the signal of features between the leak and no leak should be in the same position except the signal of leak 1 and leak 2. However, due to the time lag, the no leak signal is delayed, and results are a different distance from the leak signal. The main objective of this study is to detect the leak in the pipeline. The distance of leak one is chosen because there is no signal at no- leak data. Table 1 shows the summary data of the trial two experiments by using the galvanized iron pipe. The table shows the location of leak 1 , leak 2 and output when three conditions apply to the experiment. It can be seen that the error of the measured distance and analyzed distance is range from $0.81 \%$ to $4.26 \%$. The error for trial 2 experiment is less than the error for trial 1 experiment. Thus, it can be concluded that the noise affects accuracy when detecting the location of the leaks.

The other material used for the experiment is medium density polyethylene (MDPE) pipe. The speed of sound of this material is $950 \mathrm{~cm} / \mathrm{s}$. Table 2 shows the summary data of the trial 1 experiment by using the MDPE pipe. MDPE pipe also absorbs the signal reflected by the features and thus weaken the signal. This will lead to low signal or no signal of the features collected. Besides that, the error of the distance is quite high which are ranging from $2.88 \%$ to $16.1 \%$. Thus, it can be concluded that the accuracy of the leak location in MDPE pipe is lower compared to a galvanized iron pipe. 
Leakage Detection in Pipeline using Wavelet Transform Method

TABLE I. TABLE 1: SUMMARY EXPERIMENT DATA BY USING GALVANIZED IRON

\begin{tabular}{ccccc}
\hline Condition & Features & Measured Distance, $\mathrm{m}$ & Analyzed Distance, $\mathrm{m}$ & Error, \% \\
\hline \multirow{3}{*}{ Open Both Leak } & Leak 1 & 1.15 & 1.101 & 4.26 \\
& Leak 2 & 3.00 & 3.088 & 2.93 \\
& Output & 5.31 & 5.353 & 0.81 \\
\hline \multirow{2}{*}{ Open Leak 1 } & Leak 1 & 1.15 & 1.139 & 0.96 \\
& Leak 2 & 3.00 & - & - \\
& Output & 5.31 & 5.24 & 1.32 \\
\hline \multirow{2}{*}{ Open Leak 2 } & Leak 1 & 1.15 & 2.943 & - \\
& Leak 2 & 3.00 & 5.365 & 1.90 \\
& Output & 5.31 & & 1.04 \\
\hline
\end{tabular}

TABLE II. TABLE 2: SUMMARY EXPERIMENT DATA BY USING MDPE

\begin{tabular}{ccccc}
\hline Condition & Features & Measured Distance, $\mathrm{m}$ & Analyzed Distance, $\mathrm{m}$ & Error, \% \\
\hline \multirow{3}{*}{ Open Both Leak } & Leak 1 & 1.34 & 1.218 & 9.10 \\
& Leak 2 & 3.99 & 3.793 & 4.94 \\
& Output & 6.15 & 6.047 & 1.67 \\
\hline \multirow{2}{*}{ Open Leak 1 } & Leak 1 & 1.34 & 1.556 & 16.1 \\
& Leak 2 & 3.99 & - & - \\
& Output & 6.15 & 6.327 & 2.88 \\
\hline \multirow{2}{*}{ Open Leak 2 } & Leak 1 & 1.34 & - & - \\
& Leak 2 & $3 . .99$ & 3.762 & 5.71 \\
& Output & 6.15 & 6.359 & 3.40 \\
\hline
\end{tabular}

\section{CONCLUSION}

All the data collected, result and analysis are obtained from the experimental process by using transient analysis of waves for leakage detection. Transient is occurs when there is a disturbance in the flow of the fluid and it this study the solenoid valve is used to create the pressure transient. The wave propagated throughout the pipe and reflected when it encounters the features such as a leak, elbow, junction and many more. The signal reflected then be collected by using only one pressure transducer and in cases of this study which are using strain gauge pressure transducer and Piezotronics pressure sensor. Wavelet analysis of MATLAB is used as a method to analyze the collected data. From the result, it shows that the location of the leak can be identified and located from the peak that shows in the graph after being analyzed. The result also shows that the accuracy of the leak location is higher in the galvanized iron pipe (below than 5\% for error value) compared to the medium density polyethylene pipe (below than 20\%). Thus, it can be concluded that the type of material pipe used to affect the accuracy of the leak location. The proposed technique by using one pressure transducer predicts the location satisfactorily as it can detect the location of the leaks.

\section{ACKNOWLEDGMENT}

The authors are grateful to the Ministry of Higher Education Malaysia, which supported this research via RAGS grant RDU 121410.

\section{REFERENCES}

[1] http://www.unesco.org/education

[2] B. Brunone, "Transient test based technique for leak detection in outfall pipes", Journal of Water Resources Planning and Management. 125(5), 1999, 302-306

[3] M. Ferrante, and B. Brunone, "Pipe system diagnosis and leak detection by unsteady-state tests Wavelet analysis. Advances in Water Resources," 26, 2003, pp: 107-116.

[4] S.B.M. Beck, M.D. Curren, N.D. Sims, and R. Stanway, "Pipeline network features and leak detection by cross-correlation analysis of reflected waves," Journal of Hydraul. Eng., 131(8), 2005, pp: 715-723.

[5] I. Al-Shidhani, S.B.M. Beck, and W.J. Staszewski, "Leak monitoring in pipeline networks using wavelet analysis," Key Engineering Materials, 245-346, 2003, pp: 51-58.

[6] W. Mpesha, S.L. Gassman, and M.H. Chaudhry. "Leak detection in pipes by frequency response method," J. Hydraul. Eng., 127(2), 2001, pp: $134-147$. 
M.F Ghazali, A. K Samta

[7] G. Thakur, and H.T. Wu, Synchrosqueezing-based Recovery of Instantaneous Frequency from Nonuniform Samples, Mathematics Department, Princeton University, Princeton, NJ 08544, USA, 2011.

[8] C.Valens, "A Really Friendly Guide to Wavelets," Journal of wavelet, 1999, pp.50 - 100

[9] W.L. Bayissa, N. Haritos, and S.Thelandersson. "Vibration-Based structural damage identification using the wavelet transform," 13(8), 2007. pp. $4-6$

[10] http://homerepair.about.com/od/plumbingrepair/ss/Types-Of-Home-Piping-Materials 6.htm

E.Fisher, "New Techniques For leak detection in challenging acoustic environments," Journal of vibration research, 2007 , pp. $72-91$ 